\title{
COMMENT
}

\section{Cancer therapy with decreased SARS-CoV-2 infection rates in} cancer patients

\author{
Takuma Hayashi (iD ${ }^{1,2 \bowtie}$ and Ikuo Konishi ${ }^{1,3}$ \\ (c) The Author(s), under exclusive licence to Springer Nature Limited 2021
}

British Journal of Cancer (2022) 126:521-522; https://doi.org/10.1038/s41416-021-01685-3

\section{SUMMARY}

The mammalian target of the rapamycin (mTOR) pathway has been demonstrated to be modulated by numerous RNA viruses. Recent evidence points toward modulation of mTOR in severe acute respiratory syndrome coronavirus 2 (SARS-CoV-2) infection. Patients with cancer, who receive antineoplastic agents that suppress the mTOR/phosphoinositide 3-kinase (PI3K) signaling pathway, may have lower SARS-CoV-2 infection rates and coronavirus disease 2019 (COVID-19) severity.

COVID-19 pandemic urgently warranted the necessity to develop novel therapies against SARS-CoV-2 infection. The mammalian target of the rapamycin (mTOR) pathway has been demonstrated to be modulated in numerous RNA virus infections. Frequently. However, inhibiting mTOR results in the suppression of viral growth and replication. Recent scientific evidence points toward the modulation of mTOR in SARS-CoV-2 infection.

Foote et al. reported that according to the signatures database, 91 antineoplastic agents were associated with downregulation of the gene coding for angiotensin-converting enzyme 2 (ACE2), the entry receptor for SARS-CoV-2. These agents include mTOR/ phosphoinositide 3-kinase (PI3K) inhibitors and antimetabolites [1]. Antineoplastic agents, such as mTOR/PI3K inhibitors and antimetabolites, that inhibit the expression of ACE2 might exhibit clinical anti-SARS-CoV-2 activity [1]. Patients who received antitumor agents that could lower ACE2 showed a statistically significantly reduced SARS-CoV-2 positive rate of $7.0 \%$ compared to $12.9 \%$ of patients who received other antitumor treatments [1].

$\mathrm{Li}$ et al. demonstrated the Middle East respiratory syndrome coronavirus (MERS-CoV) receptor dipeptidyl peptidase-4 (DPP4; CD26), a well-known diabetes-related factor, as a candidate binding target of the SARS-CoV-2 spike protein [2]. Both MERSCoV and SARS-CoV-2 recognize and bind strongly to the cell receptors, DDP4 and ACE2, respectively, and efficiently infect both bronchial and bronchiole epithelial cells that are not protected by mucus barrier [3].

Remarkably, by $24 \mathrm{~h}$ time point after MERS-CoV infection, most of the activated signaling pathways were reported to be associated with cell junctions, e.g. adherens junction pathways, etc., wingless-type MMTV integration site family (Wnt), transforming growth factor (TGF) or $\mathrm{PI} 3 \mathrm{~K} /$ protein kinase $\mathrm{B}$ (AKT)/mTOR inducible signaling pathways, with the suppression of multiple innate immune response-related signaling pathways [4]. A Study of the activated signaling responses at all time points revealed that MERS-CoV infection regulates a broad range of cellular functions, including the PI3K/AKT/mTOR signaling pathways [4].

Of particular interest in the SARS-CoV-2 life cycle is its modulation of the mTOR molecule and its pathways. Importantly, the mTOR signaling pathway is necessary for viral translation during the SARS-CoV-2 life cycle [5]. To ensure the replication of coronavirus, SARS-CoV-2 infection reportedly causes activation of mTOR/PI3K signals. Hepatocyte Nuclear Factor 1 (HNF1) family, which is not included in the mTOR/PI3K signaling pathway, markedly induces the expression of $A C E 2$ gene $[6,7]$. Presumably, mTOR/PI3K signal activation may be a change in cellular function that occurs after SARS-CoV-2 infection rather than inducing ACE2 gene expression. Therefore, it seems that the rates of SARS-CoV-2 positivity and the attendant severity of COVID-19 will be lower in cancer patients who received antineoplastic agents that suppress the mTOR/PI3K signaling pathway as compared to cancer patients administered other antineoplastic agents (Fig. 1).

Recent studies have shown that a subset of the approved kinase inhibitors targeting the extracellular signal-regulated kinase (ERK)/ mitogen-activated protein kinase (MAPK) and PI3K/AKT/mTOR pathways significantly inhibited coronavirus replication, including SARSCoV-2, whether they were administered before or after the viral infection was established. In clinical settings, mTOR/PI3K inhibitors (everolimus, temsirolimus and alpelisib), other kinase inhibitors (dasatinib and crizotinib) and antimetabolites (decitabine and gemcitabine) are prescribed as antineoplastic agents to patients with cancer. Thus, antineoplastic agents inhibiting the activity of ERK/MAPK or PI3K/AKT/mTOR are not only suppressing malignant tumour growth but also mitigating the severity of cancer patients with SARSCoV-2 infection by inhibiting SARS-CoV-2 replication (Fig. 1). Patients with cancer who received antineoplastic agents that inhibit the mTOR/PI3K signaling pathway might exhibit a statistically significant reduction in SARS-CoV-2 positivity rates and correspondingly lower rates of COVID-19 severity. Taken together, from the perspective of preventing the severity of COVID-19 and treating cancer patients infected with SARS-CoV-2, it is important to consider the therapeutic effects of mTOR inhibitors, other kinase inhibitors, or DPP4 inhibitors because of their inhibitory properties on the mTOR/PI3K signaling pathway.

\footnotetext{
${ }^{1}$ National Hospital Organization, Kyoto Medical Center, Kyoto, Japan. ${ }^{2}$ START-program, Japan Science and Technology Agency (JST), Tokyo, Japan. ${ }^{3}$ Kyoto University, Graduate School of Medicine, Kyoto, Japan. ${ }^{\times}$email: yoyoyo224@hotmail.com
}

Received: 30 September 2021 Revised: 29 October 2021 Accepted: 22 December 2021

Published online: 30 December 2021 


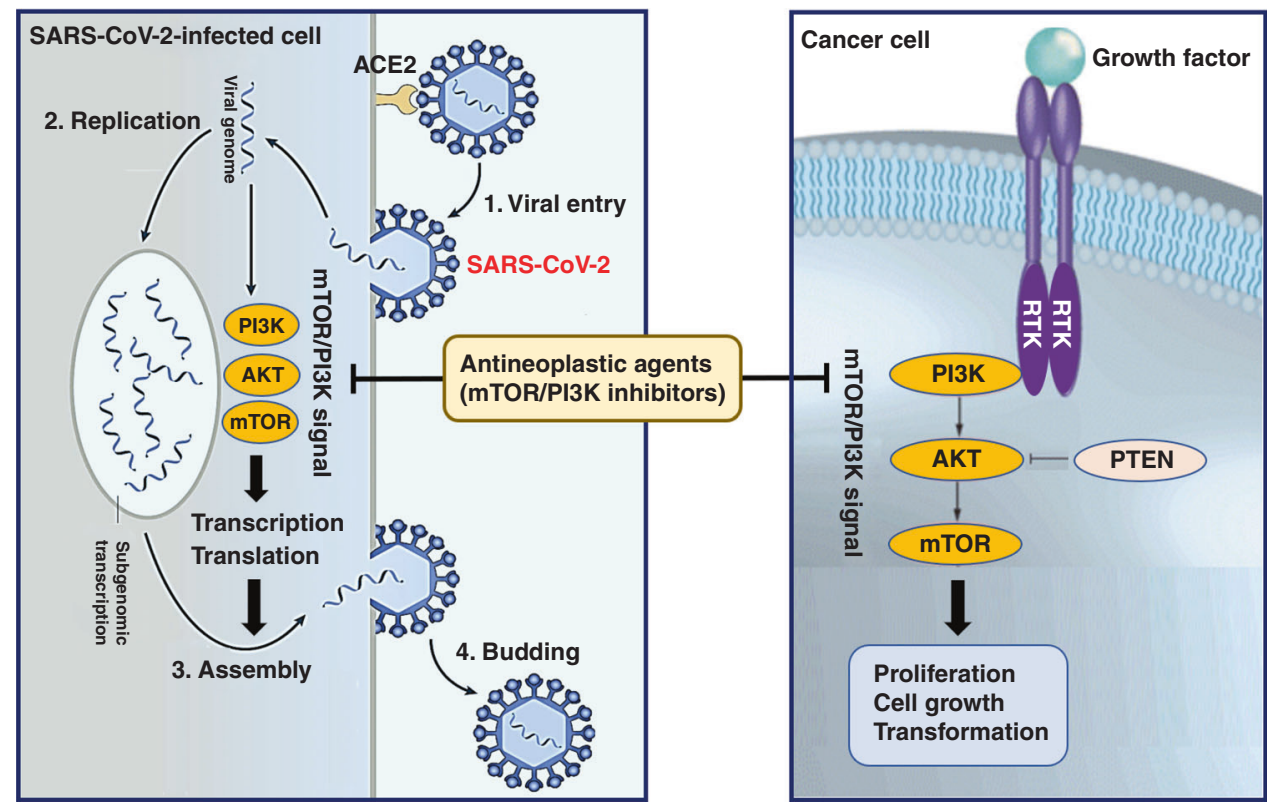

Fig. 1 Antiviral effect of antineoplastic agents inhibiting mTOR/PI3K signaling pathway in SARS-CoV-2 infection in cancer patients. Activation of the mTOR/PI3K signaling pathway in the host cell contributes to the viral life cycle from gene transcription to mRNA translation into proteins and subsequent assembly/budding of SARS-CoV-2. Additionally, mTOR/PI3K signaling pathway promotes replication and proliferation of cancer cells. Therefore, antineoplastic agents that inhibit the mTOR/PI3K pathway does not only suppress the growth of cancer cells but also inhibit the replication of SARS-CoV-2. AKT protein kinase B-alpha, mTOR mammalian target of rapamycin, PI3K phosphoinositide 3-kinase, RTK receptor tyrosine kinase, SARS-CoV-2 severe acute respiratory syndrome coronavirus 2.

\section{Disclaimer}

The material (manuscript and figure) is original research. It has not been previously published and has not been submitted for publication elsewhere while under consideration.

\section{DATA AVAILABILITY}

This manuscript is an editorial and does not contain research data. Therefore, there is no research data or information to be published or opened.

\section{REFERENCES}

1. Foote MB, White JR, Jee J, Argilés G, Wan JCM, Rousseau B, et al. Association of antineoplastic therapy with decreased SARS-CoV-2 infection rates in patients with cancer. JAMA Oncol. 2021. https://doi.org/10.1001/jamaoncol.2021.3585.

2. Li Y, Zhang Z, Yang L, Lian X, Xie Y, Li S, et al. The MERS-CoV receptor DPP4 as a candidate binding target of the SARS-CoV-2 spike. iScience. 2020;23:101160.

3. Gale P. Thermodynamic equilibrium dose-response models for MERS-CoV infection reveal a potential protective role of human lung mucus but not for SARS-CoV-2. Micro Risk Anal. 2020;16:100140.

4. Kindrachuk J, Ork B, Hart BJ, Mazur S, Holbrook MR, Frieman MB, et al. Antiviral potential of ERK/MAPK and PI3K/AKT/mTOR signaling modulation for Middle East respiratory syndrome coronavirus infection as identified by temporal kinome analysis. Antimicrob Agents Chemother. 2015;59:1088-99.

5. Karam BS, Morris RS, Bramante CT, Puskarich M, Zolfaghari EJ, Lotfi-Emran S, et al. mTOR inhibition in COVID-19: a commentary and review of efficacy in RNA viruses. J Med Virol. 2021;93:1843-6.

6. Beacon TH, Delcuve GP, Davie JR. Epigenetic regulation of ACE2, the receptor of the SARS-CoV-2 virus. Genome Apr. 2021;64:386-99.

7. Memon B, Abdelalim EM. ACE2 function in the pancreatic islet: Implications for relationship between SARS-CoV- 2 and diabetes. Acta Physiol. 2021. https://doi. org/10.1111/apha.13733

\section{ACKNOWLEDGEMENTS}

We thank all the medical staff and co-medical staff for providing and helping medical research at National Hospital Organization Kyoto Medical Center. We appreciate
Crimson Interactive Japan Co., Ltd. for revising and polishing our manuscript in the native English language.

\section{AUTHOR CONTRIBUTIONS}

$\mathrm{TH}$ wrote the manuscript. IK carefully reviewed the manuscript and commented on aspects of clinical medicine. IK shared information on clinical medicine and oversaw the entirety of the study.

\section{FUNDING INFORMATION}

This research comment was performed with the support of the following research funding: Japan Society for the Promotion of Science for TH (Grant No. 19K09840), START-program Japan Science and Technology Agency (JST) for TH (grant No. STSC20001) and National Hospital Organization Multicenter clinical study for TH (grant No. 2019-Cancer in general-02).

\section{COMPETING INTERESTS}

The authors declare no competing interests.

\section{ADDITIONAL INFORMATION}

Supplementary information The online version contains supplementary material available at https://doi.org/10.1038/s41416-021-01685-3.

Correspondence and requests for materials should be addressed to Takuma Hayashi.

Reprints and permission information is available at http://www.nature.com/ reprints

Publisher's note Springer Nature remains neutral with regard to jurisdictional claims in published maps and institutional affiliations. 